\title{
Endomicroscopy for Computer and Robot Assisted Intervention
}

\author{
Siyang Zuo, and Guang-Zhong Yang, Fellow, IEEE
}

\begin{abstract}
Endomicroscopy is a new technique that allows human tissue to be characterized in vivo, and in situ, circumventing the need for conventional biopsy and histology. Despite increased application and growing research interests in this area, the clinical application of endomicroscopy, however, is limited by difficulties in ergonomic control, consistent probe-tissue contact, large area surveillance and retargeting. Recently, advances in high-speed imaging, mosaicing and robotics have aimed to address these difficulties. The development of robot-assisted devices in particular has shown great promises in extending the clinical potential of endomicroscopy. Issues related to miniaturization, adaptation to tissue deformation, control stability, force and position compensation, cost and sterility are being pursued by both research and commercial communities. In this paper, recent clinical and technical developments in different aspects of computer and robotic assisted endomicroscopy interventions including instrumentation, multiscale integration, and high-speed imaging techniques are presented. We further address emerging trends and new research opportunities towards more widespread clinical acceptance of robotically assisted endomicroscopy technologies.
\end{abstract}

Index Terms-Endomicroscopy, Minimally Invasive Surgery, Image mosaicing, Surgical robot, Robotic assisted intervention.

\section{INTRODUCTION}

\section{A. Background of endomicroscopy}

Spectroscopic methods such as diffuse reflectance, Raman and fluorescence spectroscopy are useful for in vivo assessment of cancer, atherosclerosis and ischemia. Current techniques under development include visible diffuse light spectroscopy applied in the colon to detect colonic ischemia [1], the use of fluorescence spectroscopy to detect atherosclerotic lesions [2] and the use of Raman sensing to detect cervical cancer [3]. There has been increasing focus in recent years on simultaneous large area surveillance with high spectral resolution, and thus higher sensitivity and specificity, potentially with multiple labels and multimodal spectroscopy.

Over the last decade, endomicroscopy has shown to be a promising tool for tissue characterization compared to traditional biopsy. Instead of taking a number of biopsies, and examining these with histology, an optical probe can be

This work was supported by EPSRC grant EP/IO27769/1: SMART Endomicroscopy. S. Zuo is with the Key Laboratory of Mechanism Theory and Equipment Design of Ministry of Education, Tianjin University, Tianjin 300072, China (email: siyang_zuo@tju.edu.cn). G.-Z. Yang are with the Hamlyn Centre for Robotic Surgery, Imperial College London, London SW7 2AZ, U.K. (e-mail: g.z.yang@imperial.ac.uk). inserted into the patient and used to obtain high resolution images of tissue in real time. There are several advantages to this approach. Firstly, it is well suited to serial examination of conditions since the process does not involve damage to the tissue. Secondly, diagnosis is available in real-time rather than after a period of a day or more. Thirdly, it could obtain dynamic in vivo information such as the observation of blood flowing, and the elimination of artefacts from histological fixation and preparation. Additionally, it could also be potentially incorporated into the workflow during therapeutics such as tissue ablation/excision to confirm the edges of the lesion. Finally, the surgeon can examine an area as large as is required, rather than relying on a small area. These advantages make endomicroscopy particularly attractive to Minimally Invasive Surgery (MIS).

One promising technique is confocal fluorescence laser endomicroscopy (CLE), which provides high-resolution, real-time, in vivo, and in situ imaging at cellular and subcellular levels [4-8]. Originally proposed by Marvin Minsky, the concept of confocal microscopy allows high-resolution, blur free fluorescence microscopy and immunohistochemistry on excised tissues [9]. More recently, miniaturization of the confocal laser scanning microscope has enabled it to be used as an in vivo device to provide virtual histology. In 2004, confocal laser endomicroscopy was first introduced, allowing the surgeon to obtain real time in vivo optical biopsies with endoscopy [10]. Subsequent developments include probe based confocal fluorescence laser endomicroscopy (pCLE) [11-13] using fibre bundles combined with miniaturized laser scanners, fluorescence endomicroscopy [14] and white light microscopy [15]. These techniques generate images of the tissue surface at resolutions of 1-2 micrometers and with a field-of-view of typically 500 micrometers. Most of these techniques rely on the use of either intravenous or topical contrast agents [16] (such as fluorescein or methylene blue) in order to enhance contrast. In some cases, the exogenous contrast agents are not required, such as in the lung since there is endogenous auto-fluorescence of the elastin within the endobronchial and the alveolar septal walls. Real-time confocal endomicroscopic image acquisition of in vivo tissue morphology is achieved through the process of optical sectioning which involves point-by-point illumination of the tissue surface using a rapidly scanning laser. Endomicroscopy has been used extensively in the gastrointestinal tract, most notably during endoscopic surveillance of Barrett's esophagus [17].A related technique, optical coherence tomography (OCT) [18] can generate cross-sectional images of tissue. OCT relies only on differences in reflectivity to generate contrast. It was originally developed for ophthalmology, but now has been extended to endoscopic 
applications such as those for the gastrointestinal tract $[19,20]$ and endovascular intervention $[21,22]$. Tethered capsule OCT [23] is currently being commercialized for large area imaging of the esophagus, and reaching into the duodenum [24]. The NvisionVLE imaging system allows clinicians to evaluate the esophageal tissue microstructure in unprecedented details during a standard endoscopic procedure [25]. From bench-top microscopes [26, 27], the platforms are progressing toward smarter and smaller systems, with exciting research conducted on millimeter-scale devices (Fig. 1).

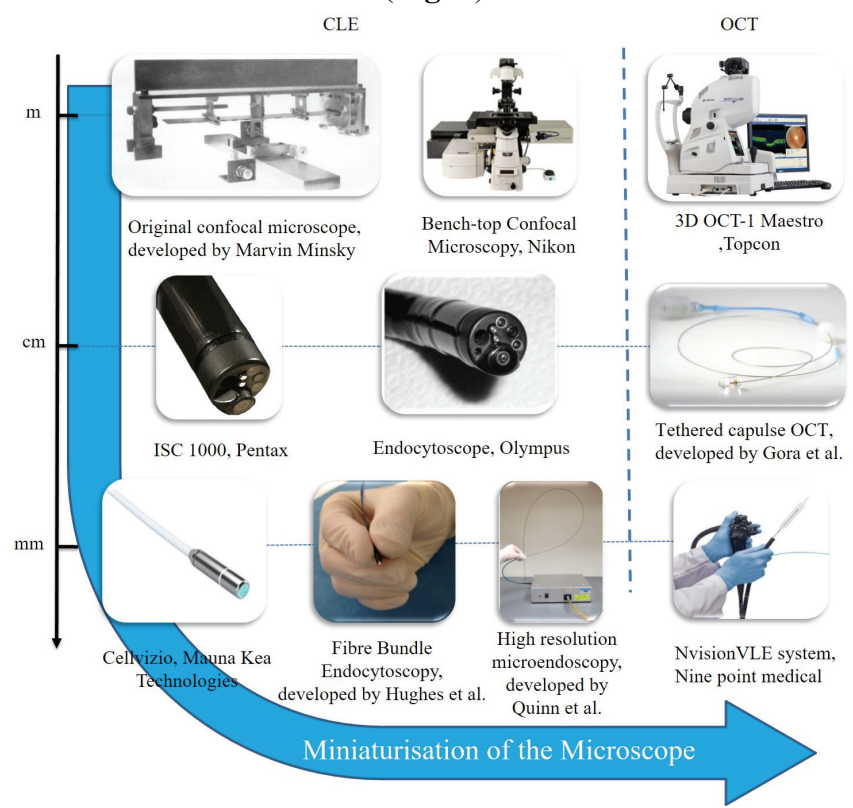

Fig. 1 Miniaturisation of microscope, "Original confocal microscope" image: adapted from [9], (C) Wiley-Liss, Inc.; "Bench-top confocal microscopy" image: adapted from [26], (C) Nikon instruments, Inc.; "3D OCT-1 Maestro" image: adapted from [27], (C) Topcon, Inc.; "ISC 1000" image: adapted from [50], (C) Pentax, Inc.; "Endocytoscope" image: adapted from [52], (C) Olympus medical, Inc.; "Tethered capulse OCT" image: adapted from [23], (C) Nature group publications; "Cellvizio" image: adapted from [51], (C) Mauna Kea Technologies, Inc.; "Fibre bundle endocytoscopy" image: adapted from [54], (C) osapublishing.org; "High resolution microendoscopy" image: adapted from [53], (C) journals.plos.org publications; "NvisionVLE system" image: adapted from [25], (C) Nine point medical, Inc.

All of these techniques, however, suffer from the limitation of only providing a small probe region whilst the organs of interest have a large surface area to be surveyed. For pCLE, recent clinical evaluation of the system revealed relatively low sensitivity and positive predictive value of 'optical biopsy'. Moreover, its overall accuracy is inadequate in many settings to obviate the need for conventional biopsy; for example, it cannot sufficiently reassure the colonoscopist over hyperplastic-looking polyps to avoid pinch biopsy of any colonic polyps. Also, it is difficult to make standard four quadrant biopsy of Barrett's oesophagus redundant. In addition to difficulties in ergonomic control, articulation and manipulation of these miniprobes make the performance of large area surveillance and repeated examination challenging.
All these problems are further complicated by tissue deformation and difficulties in manually maintaining consistent probe contact during examinations.

\section{B. Endomicroscopy in MIS}

With the development of MIS in recent years, providing in situ, in vivo morphological and functional imaging of the exposed tissue surface has become an important research topic. This can further benefit MIS in providing tissue detection, labelling, and targeting both at macro and cellular levels. Clinical application of endomicroscopy represents a promising modality that allows real-time, high resolution imaging of tissues in vivo. It generates an 'optical biopsy' without conferring tissue trauma. For in vivo application of endomicroscopy for MIS, a number of groups have investigated the use of confocal endomicroscopy, which following the application of a contrast agent can allow the detection of disruption in the pit pattern in the colon wall, reveal microvasculature in sessile polyps, and highlight intestinal metaplasia in Barrett's Oesophagus [28, 29]. Endomicroscopy is also used for the assessment of indeterminate biliary strictures [30,31], and distal segments of the lung through to alveoli [32]. It is comparable to colonoscopic histopathology in diagnosing colorectal cancer, and is better in conjunction with conventional endoscopy [33].

The technique has also the potential to be used in conjunction with fluorescence molecular imaging [34, 35]. Another promising approach is to use fluorophore labelled antibodies to label specific molecules expressed by the lesion that is being optically biopsied. Also of relevance is the potential for labelling endogenous ligands with fluorescent tags for the evaluation of specific receptors [36]. For assessing micro-circulation, endomicroscopy has been used to examine morphometry, capillary permeability, functional capillary density, and vasoconstriction and dilation effects. In addition, the possibility to visualize fluorescent red blood cells or leukocytes has also been evaluated [37]. There are also new clinical applications in breast conserving surgery [38-41].

With the use of miniprobes for MIS, a range of opportunities can be explored. The first is in situations where a tissue biopsy would be contraindicated like the examination of an intestinal anastomosis. In an acute setting, an indication of blood supply to the anastomosis is invaluable to the decision of whether to re-fashion a dusky looking anastomosis or bring out stomas. A histological biopsy of an anastomosis will not provide this information, whereas a real-time examination of the blood supply with systematic and direct visualization of the arterioles and capillary beds can provide data that can significantly aid in the decision making process. An equally useful scenario is in the assessment of ischemic bowel for determining whether to resect or not. This relatively common scenario still relies heavily on macroscopic examination of the bowel segment.

In surgical scenarios when histological tissue biopsy is possible but associated with higher morbidity, the use of probe-based imaging during MIS is particularly important. An example of this is lymph node or tissue biopsy in mediastinum, retroperitoneum or para-aortic space, such as subcarinal node sampling during mediastinoscopy, where the trauma of biopsy 
can result in potentially fatal bleeding in anatomical regions which are only accessible through a median sternotomy or thoracotomy. In these circumstances, the benefits of 'optical biopsy' in avoiding the risks of bleeding or nerve injury compared to the use of a conventional biopsy can be significant. The same principle applies to biopsy sites where biopsy trauma may cause unacceptable damage such as in the eyes or brain. As our understanding of cell-specific molecules increases, we are likely to be able to use molecular markers to explicitly identify tissue types and cell lineages in real-time [42]. This can have significant implications for pre-operative cancer staging as well as resectional cancer surgery. In pre-operative colorectal cancer staging, for instance, existing techniques such as CT imaging are suboptimal at identifying nodal status [43] and systematic optical biopsies of the entire colonic lymph node field with targeted cancer cell molecular markers have the ability to ensure optimum and personalized treatment for individual patients. In resectional surgery when small margins are required, such as in neurosurgery, it is imperative to achieve a complete (R0) resection whilst at the same time leaving as much healthy tissue as possible. In the resection of brain tumors, contrast imaging has already been used to achieve more accurate resection margins [44] and microscopic evaluation of tumor deposit in real-time not only is likely to greatly improve the accuracy of resection, and therefore prognosis, but also minimize the number of further interventions necessary.

\section{Technical Challenges}

Despite aforementioned promises, endomicroscopic probes are still in their infancy and there are many technical problems that need to be overcome before they can realize their full clinical potential. In practice, the deployment of miniprobes for large area tissue scanning presents significant challenges relating to precision, accuracy and economy of tip scanning motion. Currently, the field-of-view of the images obtained is less than $1 \mathrm{~mm}$ in diameter and this could be as low as $0.3 \mathrm{~mm}$ in diameter when high-resolution confocal miniprobes are used. It is unequivocal that the information obtained from a static miniprobe will not be truly representative of the corresponding tissue surface as adjacently placed cancer cells could easily be missed if they are not brought to the surgeon's view. A potential solution to this is to increase the field-of-view obtained by stitching adjacent image frames together as the probe is moved across the tissue surface, a technique known as mosaicing [45-49]. However, this is often difficult to translate the probe tip over the tissue in a smooth and precisely controlled fashion with conventional controls of endoscope. There is further challenging due to the relatively large tissue surface area that needs to be assessed, which is a time-consuming process and inevitably induces fatigue to the surgeon. In practice, this could lengthen the operation time, which in turn increases the risk of anaesthetic complications to the patient and reduces operating productivity. Moreover, the resolution is limited due to the individual element size of the fibre bundle array. Other limitations of the current systems and technical challenges also include insufficient localization, making intra-operative anatomical and functional co-registration and identification of previously visited biopsy sites or serial screening following therapeutic procedures difficult. There is also a lack of effective visualization of multi-scale integration/mosaicing of functional data for linking point based 'optical biopsy' to regional or large area tissue characterization and mapping.

\section{Overview of Technical Developments}

The purpose of this review is to present the emerging trends and opportunities for computer and robotic assisted interventions with endomicroscopy. Whilst commercial organizations are focusing on improved instrumentation and probe design, research groups have turned towards enhanced imaging processing and more recently robotic actuation of the devices. In our study, search strategy includes three steps that are (i) search database, (ii) review title and abstract, and (iii) review the full text of the paper. We mainly highlight the work after 2005. Table 1 lists the main topics to be addressed in this review, illustrating the key features and current and future research trends in the field of computer and robot assisted interventions for endomicroscopy.

\section{INSTRUMENTATION}

For endomicroscopy, commercial platforms typically include a miniature objective lens with scanning mirrors mounted onto the distal end of an endoscope (e.g. Pentax/Optiscan, Japan [50], also called endoscopic confocal laser endomicroscopy (eCLE)), a coherent fibre-bundle with external laser optoelectronics (e.g. pCLE of Mauna Kea/Cellvizio, France [51]) by using each fibre as its own pinhole, and a white light microscopy (e.g. Olympus/Endocytoscopy, Japan [52]). The features of these commercial systems are shown in Table 2. With pCLE the focal plane depth is generally fixed for each probe, whereas with eCLE, the focal plane depth (z-axis) can be advanced between $0-250 \mu \mathrm{m}$ with $4 \mu \mathrm{m}$ resolution. The main advantage of the fibre-based system is its ease of integration with endoscopes by making use of the instrument channel, but it has relatively inferior resolution. However, their small size provides better in vivo accessibility and they are sufficiently versatile to be used with most endoscopes including cholangioscopes and ureterosocopes. pCLE probes also have a faster frame rate, thus more immune to tissue movement.

For research platforms, Quinn et al. developed a high-resolution microendoscopy for the detection of cervical neoplasia in low-resource settings [53]. It can be used to interrogate clinically suspicious areas with subcellular spatial resolution, revealing changes in nuclear to cytoplasmic area ratio. A low-cost white light endomicroscopy techniques and high frame rate endomicroscopy were developed by [54-56]. An alternative design that instead relays images to an external camera via a coherent fiber bundle has been developed. One advantage of this approach is the ease with which other compatible imaging modalities can be deployed simultaneously. They show this by acquiring quasi-simultaneous endocytoscopy and fluorescence confocal endomicroscopy images through a single fiber bundle [54]. Recently, Hughes et al [55] proposed an approach that is specific to widefield endomicroscopy in which light is injected into a leached fiber bundle near the distal end, thereby avoiding reflections from the proximal face. They use this method to demonstrate color 
TABLE 1. Overview of topics and technical trends addressed in this review.

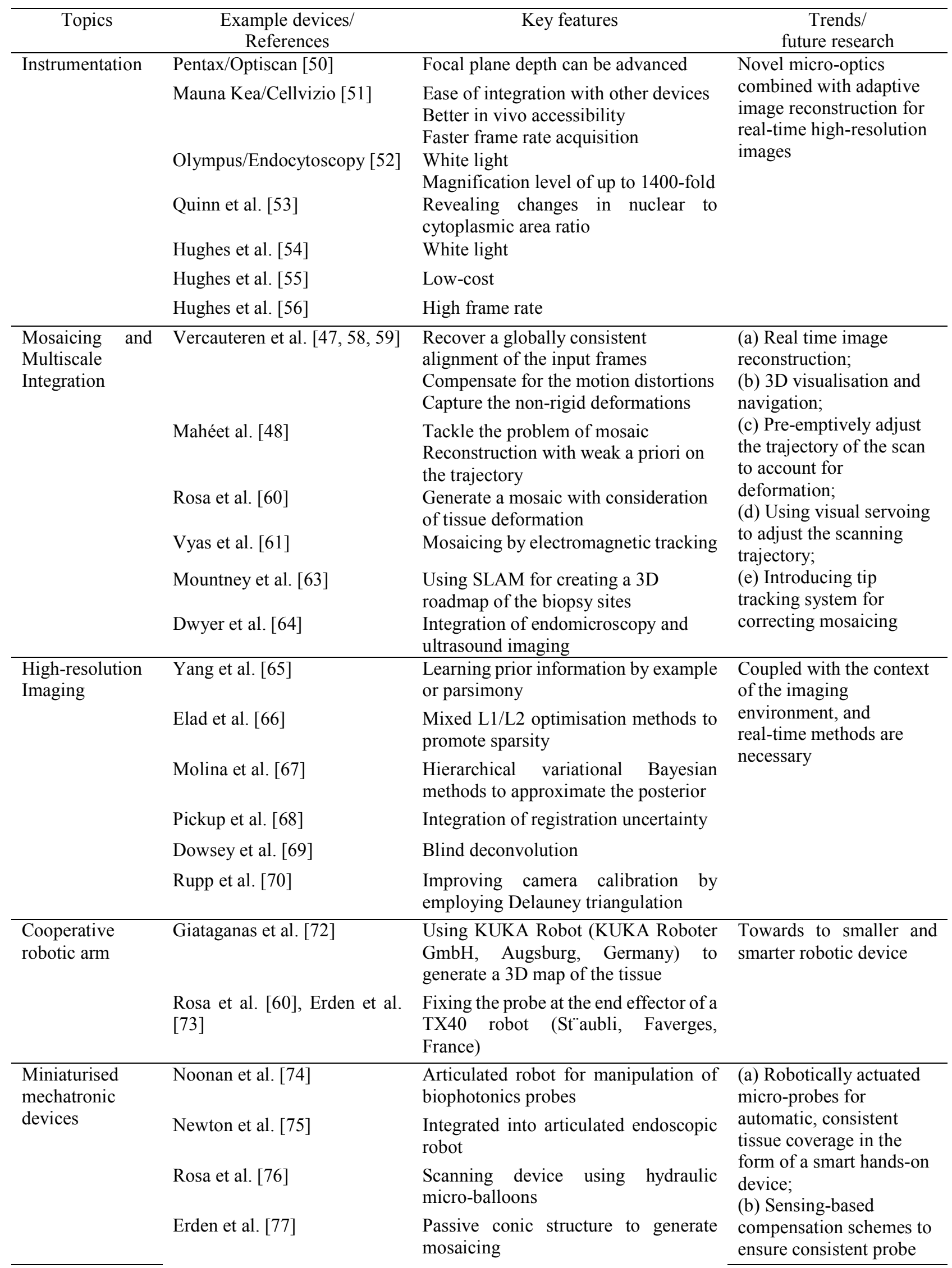


Dwyer et al. [64]

Zuo et al. [79]

Zuo et al. [80]

Noonan et al. [87]

Giataganas et al. [88]

Latt et al. [89, 90]

Gilbertson et al. [91]

Ang et al. [92], Becker et al. [93], Maclachlan et al. [94]

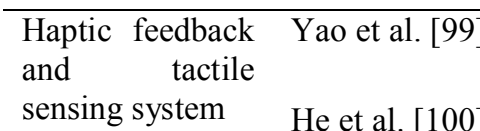

Payne et al. [101]

Stetten et al. [102]

Robotic instrument for the fusion of endomicroscopy and ultrasound

Gear-based rigid concentric tube scanning mechanism

Balloon-based linkage scanning mechanism for breast concerving surgery

LWPR control strategy and impedance control [87] approaches

Force adaptive with an embedded 6-axis force/torque sensor (ATI Nano17)

Force-contact probe for integration with surgical robot platforms(da Vinci)

Embedded force sensing into imaging probe

Incorporate intelligent force control schemes for imaging probe

Incorporating tremor cancellation by using piezoelectric actuators combined with visual servoing

Sensing the tool-tissue interactions with an accelerometer

Micro-scale force sensing based on the fiber Bragg grating (FBG)

Amplified forces onto the operator's fingertip

No need of being affixed to the ground control and force stability

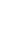

(a) Combining force control systems to imaging techniques;

(b) Novel tracking techniques to improve robustness;

(c) Techniques to improve robustness;

(d) MEMS-based inertial sensors

(a) High sensitivity;

(b) Integrated force

sensing and feedback with ungrounded force amplification;

(c) Improve robustness;

(d) Multi-DoF force and tactile feedback

TABLE 2. Features of three available commercial confocal endomicroscopy systems.

\begin{tabular}{cccccc}
\hline & eCLE & \multicolumn{2}{c}{ pCLE } & \multicolumn{2}{c}{ Endocytoscopy } \\
\cline { 3 - 7 } & & Gastro/Coloflex & Gastro/Coloflex UHD & XEC-300 & XGIF-Q260EC1 \\
\hline Company & Pentax & \multicolumn{2}{c}{ Mauna Kea } & \multicolumn{2}{c}{ Olympus } \\
\hline Depth of imaging $(\mu \mathrm{m})$ & $0-250$ & $70-130$ & $55-65$ & $0-30$ & $0-50$ \\
\hline Field of view $(\mu \mathrm{m})$ & $475 \times 475$ & 600 & 240 & $300 \times 300$ & $400 \times 400$ \\
\hline Lateral resolution $(\mu \mathrm{m})$ & 0.7 & 3.5 & 2.5 & 3.2 & 1.0 \\
\hline Diameter $(\mathrm{mm})$ & 12.8 & 2.7 & & & 3.2 \\
\hline
\end{tabular}

widefield reflectance endomicroscopy. In [56], they further demonstrate that by using a high speed linear CCD camera, line-scanning confocal endomicroscopy can achieve a frame rate of $120 \mathrm{fps}$ while maintaining sufficient resolution and signal-to-noise ratio to allow imaging of topically stained gastrointestinal tissues. This leads to improved performance of the mosaicing algorithm when compared to lower frame-rate systems. Current research focus is directing towards the design of novel optics combined with adaptive image reconstruction for real-time high-resolution images of tissue micro-structure or low-cost probes for developing countries [57].

\section{VISUALISATION TECHNIQUES}

\section{A. Mosaicing and Multiscale Integration}

Initial clinical application has highlighted the importance of wide field-of-view (FOV) coverage through video mosaicing to enable from point-based 'optical biopsy' to area to region analysis. Thus far, extensive research has been carried out in using both linear and non-linear registration techniques for simultaneous view-expansion and tissue/probe motion recovery $[47,48,58,59]$. This approach is based on a hierarchical framework that is able to recover a globally consistent alignment of the input frames, to compensate for motion distortion and capture non-rigid deformation.

For image processing in [12], in the calibration stage, an image of a uniform target is acquired, and a circular Hough transform is used to locate the fibre core centres. Since the proximal end of the fibre is fixed, these do not move over time. A Delaunay triangulation is then performed over the core centres, allowing each pixel in a defined reconstruction grid to be associated with an enclosing triangle of three fibre cores. In the reconstruction phase, the intensity of each fibre core is 


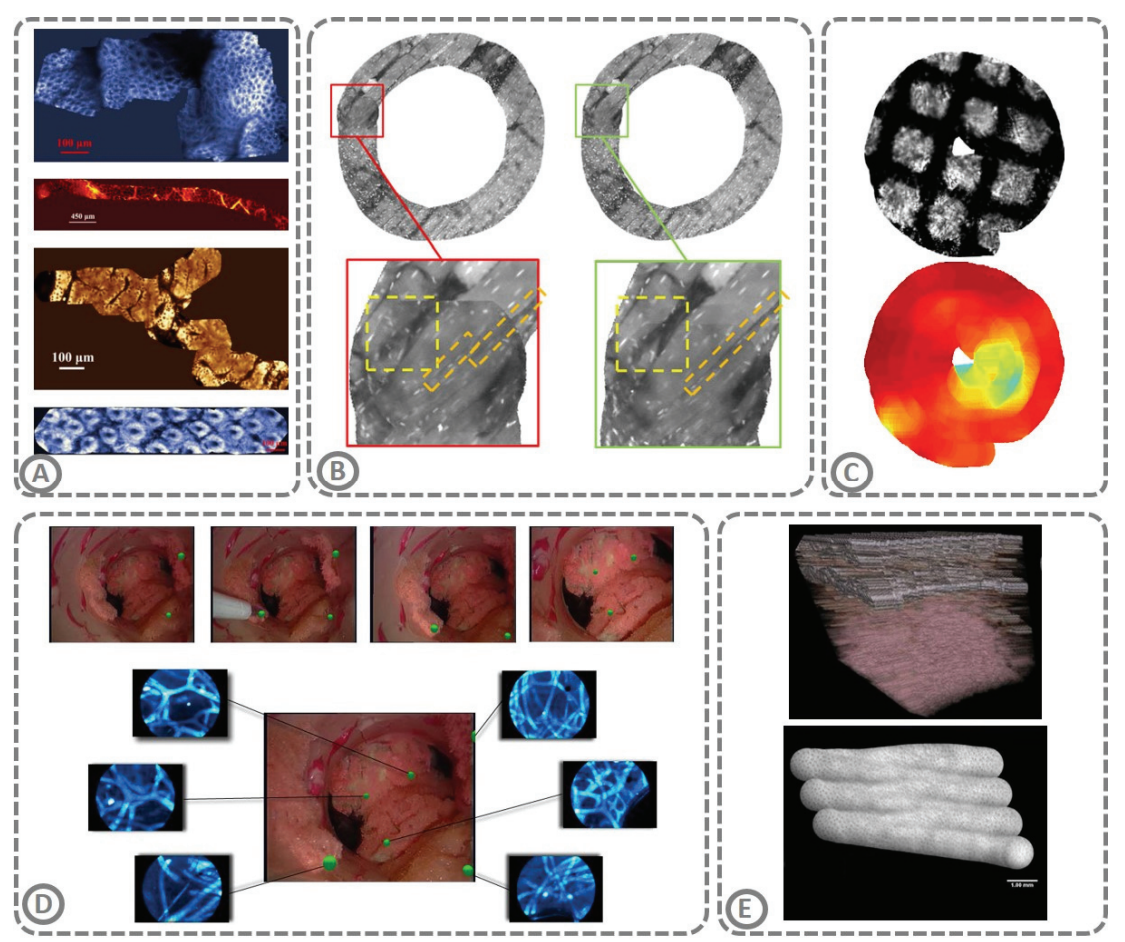

Fig. 2. Mosaicing and Multiscale Integration, (A) Robust mosaicing with correction of motion distortions and tissue deformations: adapted from [47], (C) Elsevier 2006. All Rights Reserved; (B) Mosaics of a sample of chicken breast obtained under visual servoing with a desired circular image trajectory: reprinted with IEEE permission from [60]; (C) A Viterbi approach to topology inference for large scale endomicroscopy video mosaicking: adapted from [48], C Springer-Verlag Berlin Heidelberg 2009. All Rights Reserved; (D) Optical biopsy mapping for minimally invasive cancer screening: adapted from [63], (C) Springer-Verlag Berlin Heidelberg 2009. All Rights Reserved; (E) Ex vivo imaging of porcine colon tissue imaging results: reprinted with IEEE permission from [64].

extracted by applying a Gaussian spatial filter and then taking the pixel value at the core location. The value of each pixel in the reconstructed image is then obtained by triangular linear interpolation between the values from the surrounding three cores. This algorithm would be suitable for real-time applications. The generation of mosaics in $[12,62]$ is achieved by adapting a standard method whereby the cross-correlation is calculated between successive pairs of image frames and the peak of the cross correlation is taken as an estimate of the inter-frame shift (essentially giving the instantaneous velocity). While this simple approach is useful for small mosaics, when attempting to perform larger scan there are inevitable points where tissue contact is lost, or there are insufficient image features for registration, and the algorithm fails. However, the advantage of using a mechanical scanning device is that the tangential velocity of the probe tip is approximately constant.

Mahé et al. described a framework aiming to reconstruct a mosaic of endomicroscopic images acquired via a robotized spiral trajectory [48]. First, they infer the topology of the frames that is the map of neighbors for every frame in the spiral. For this, they use a Viterbi algorithm considering every new acquired frame in the current branch of the spiral as an observation and the index of the best neighboring frame from the previous branch as the underlying state. Second, the estimated transformation between each spatial pair previously found is assessed. Reconstruction results were improved compared to reconstruction where only registration between successive frames was used.
In practice, tissue deformation is a significant limiting factor to the generation of large mosaics. For in vivo applications, this is unavoidable, as any contact based imaging method will result in tissue deformation when the probe is driven across the tissue. Rosa et al. have used a cooperative robotic arm to generate a mosaic with consideration of tissue deformation [60]. Furthermore, electromagnetic tracking has been used to enhance mosaicing [61]. Correction of distortions due to tissue deformation may also be achieved using a more sophisticated mosaicing algorithm which does not assume rigid transformations [47]. However, these algorithms are unlikely to be suitable for real-time image reconstruction [62] but nevertheless can be used for retrospective analysis.

Placing the 'optical biopsy' information within the context of the standard endoscope image is essential for the interpretation of the data to allow for subsequent retargeting for follow-up examination. Study on using Simultaneous Localization and Mapping (SLAM) with probe tracking for creating a 3D roadmap of the biopsy sites has highlighted the need for effective fusion and abstraction of the high-dimensional functional information with white light endoscopy for improved clinical acceptance [63]. Dwyer et al. developed a miniaturized robotic probe which integrates endomicroscopy and ultrasound imaging [64]. This results in the ability to perform large area mosaics coupled with ultrasound scanning. In addition, the use of endoscopic tracking is demonstrated, allowing three-dimensional 
reconstruction of the ultrasound data displayed onto the endoscopic view. Some examples of mosaicing and multiscale Integration are presented in Fig. 2.

\section{B. High-resolution Imaging}

When there is a physical or practical barrier to resolution, computational based super-resolution has been shown in a number of modalities to be effective. This is achieved by utilizing the underlying information content in overlapping acquisitions. This needs to be differentiated from optical high-resolution methods such as structured-illumination and stimulated emission depletion microscopy, which still remain as benchtop research platforms. Among existing computational super-resolution methods, the most promising developments include: learning prior information by example or via parsimony in some basis [65]; mixed L1/L2 optimisation methods to promote this sparsity [66]; hierarchical variational Bayesian methods to approximate the posterior, providing hyper-parameter estimation and guiding optimisation under uncertainty [67]; integration of registration uncertainty [68] or blind deconvolution [69]. In fibre endomicroscopy, work has been limited to improving camera calibration by employing Delauney triangulation based interpolation (with no deblurring step) on test patterns [70]. They present the two comb structure algorithms and the spatial super resolution model to simulate fiberscopic transmission for evaluation. Current approaches are rarely coupled with the context of the imaging environment, and real-time methods are necessarily restrictive [71]. It discuss a fast dynamic image fusion method for the translational motion model, assuming regular monochromatic images, considering both causal and non-causal modes.

\section{ROBOTIC ASSISTANCE}

\section{A. Cooperative robotic arm}

One of the major limiting factors of probes for in vivo use is control and manipulation of the device. Furthermore, this is an important issue since the precision of the probe trajectory can significantly influence the quality of the mosaicing results. Robotically controlled motion has the potential to provide high precision for generating mosaics.

Several research groups focused their studied on high-accuracy industrial robot assisted scanning (Fig. 3). A cooperative robotic arm (KUKA Roboter GmbH, Augsburg, Germany) has been used to generate a 3D map of the tissue [72]. The system provides a hands-on, learning based framework for optimal trajectory coverage from surgeon demonstrated motions. The position and force information acquired during the scanning are also used to simultaneously reconstruct the surface morphology and combined with the pCLE images to generate a 3D functional map of the tissue. Larger mosaics were also achieved by [60] using a TX40 robot (St“aubli, Faverges, France). Erden et al. also used TX40 robot to demonstrate superior performance of the spiral scan over the raster [73].

The limitation of using cooperative robotic arm is the difficulty of adapting to clinical use due to the complex robotic arm used. Smaller and smarter robotic devices are more suitable for clinical use, as well as to meet the sterilisation requirements.

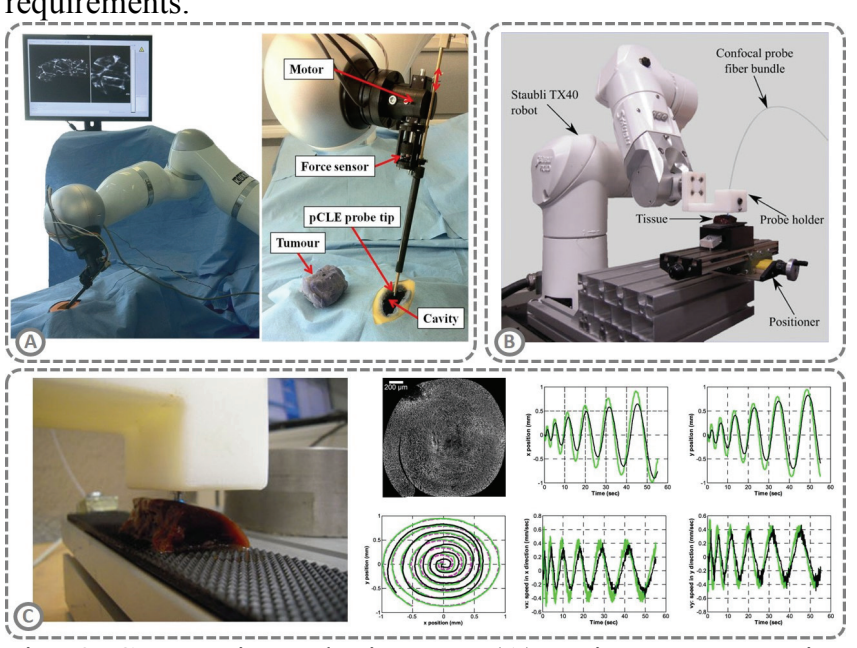

Fig. 3 Cooperative robotic arm, (A) Using a cooperative robotic arm to generate a $3 \mathrm{D}$ map of the tissue: reprinted with IEEE permission from [72]; (B) Larger mosaics achieved by using a TX40 robot: reprinted with IEEE permission from [60]; (C) Using TX40 robot to demonstrate superior performance of the spiral scan: reprinted with IEEE permission from [73].

\section{B. Miniaturized mechatronic devices}

The general limitations of cooperative robotic arms are well-recognized and have motivated the development of miniaturized mechanical scanning devices to facilitate smooth and consistent translation of the miniprobe tip against the tissue surface. The existing probe requires perpendicular apposition to the tissue with the correct pressure, which is generally achieved by using a simple short transparent mucosectomy cap on the endoscope tip to aid stabilization and targeting, but at a potential risk of inadvertent tissue damage. Furthermore, surface secretions can impair the image view and the instrument has to make contact with tissue for effective function. Development of robotically actuated micro-probes for automatic, consistent tissue coverage in the form of a smart hands-on device has clear clinical advantages. Some examples of miniaturized mechatronic devices are presented in Fig. 4.

An articulated robot for manipulation of biophotonics probes was first developed by Noonan et al. for intra-operative integration of laparoscopic instruments [74]. The device consists of a long, rigid shaft with a robotically controlled distal tip featuring three degrees of in plane articulation and one degree of rotational freedom. By using controlled joint actuation to trace overlapping trajectories, the device allows effective imaging of a larger field-of-view than a traditional dual-mode laparoscope. Newton et al. integrated a $100 \mu \mathrm{m}$ confocal depth pCLE probe into an articulated endoscopic robot (i-snake) [75]. This system has been evaluated transvaginally in a pig, under laparoscopic vision. Rosa et al. developed a scanning device using hydraulic micro-balloons [76]. The device features hydraulic actuation of the probe, along with a stabilization mechanism that solves the problem of physiological organ motion. The efficacy of the probe actuation was proven in an in vivo animal experiment, leading to the mosaic shown in Fig. 4C. This mosaic was obtained by manually controlling, in open loop, the probe motion through 


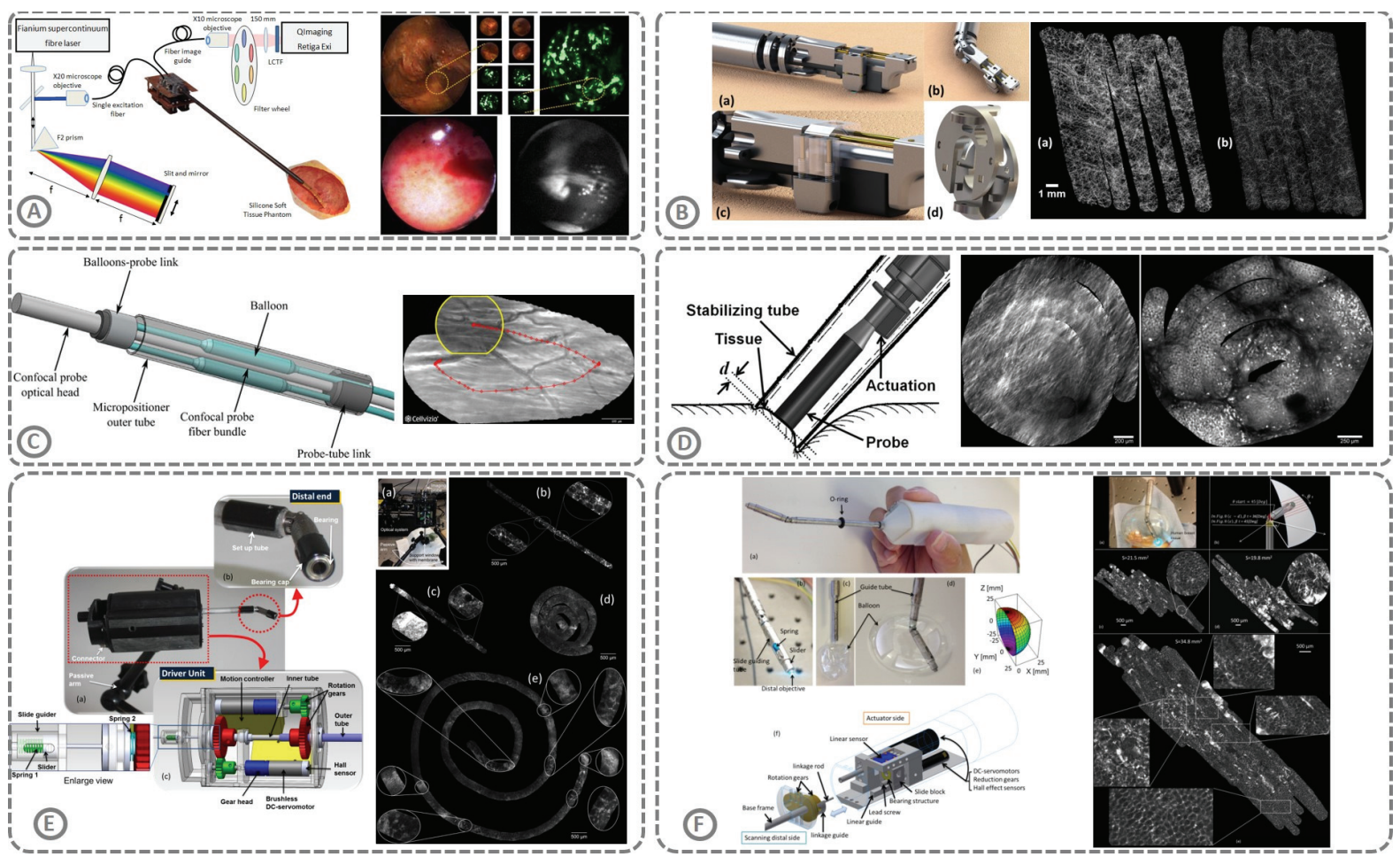

Fig. 4 Miniaturised mechatronic devices, (A) An articulated robot for manipulation of biophotonics probes: reprinted with IEEE permission from [74]; (B) Computer rendering of the scanning device with mosaicing results: reprinted with IEEE permission from [64]; (C) A scanning device using hydraulic micro-balloons: reprinted with IEEE permission from [76]; (D) A distal scanner using the conic-spiraleur mechanism: reprinted with IEEE permission from [77]; (E) A rigid concentric tube scanning mechanism to facilitate large area mosaicking: reprinted with IEEE permission from [79]; (F) A miniature linkage scanning device for microscopic imaging of the walls of the cavity created during breast conserving surgery: adapted from [80], (C) Springer-Verlag Berlin Heidelberg 2015. All Rights Reserved.

the hydraulic actuation. It was not possible to obtain larger mosaics with this mode of operation because of the difficulty of controlling the probe motion in open loop.

Erden et al. presents a mechanical design of a distal scanner using the conic-spiraleur mechanism, to perform automated spiral scan with the probe in order to construct a mosaic-image [77]. The design of the conic-spiraleur is based on using a conic structure with a particularly curved surface. The device can be inserted through a conventional 5-mm diameter trocar. The system is tested in vivo in a porcine study. They present an in vivo field of view with $3 \mathrm{~mm}^{2}$ and high resolution $(1.4-\mu \mathrm{m}$ lateral and $10-\mu \mathrm{m}$ axial) images in confocal microscopy. The target of [76] and [77] were applications for which the scanning device must remain compact. The system used in [77] was targeted towards generation a continuous mosaic of 3 $\mathrm{mm}^{2}$.

A robotic scanning endomicroscopy device has been developed for large area imaging [78]. The device uses a rigid concentric tube scanning mechanism to facilitate large area mosaicing. It has a compact design with a diameter of $6 \mathrm{~mm}$, incorporating a central channel with a diameter of $3 \mathrm{~mm}$ for passing through a fibre bundle probe. A bespoke bearing and a passive linear structure are used to control image rotation and ensure consistent tool-tissue contact. The probe tip can achieve a spiral trajectory and maintain a stable maximum acceptable linear velocity for efficient scanning by control the motors. The scanning device achieved large area spiral mosaics of human breast cancer tissues [79]. Moreover, a miniature linkage scanning device has also been developed for microscopic imaging of the walls of the cavity created during breast conserving surgery [80]. It is envisaged that this scanning device would be inserted through the incision created during BCS, and deployed against the cavity walls to allow for smooth and seamless image acquisition over a large tissue surface area. It has a compact design, with an outer diameter of $4 \mathrm{~mm}$ and a working channel of $2.2 \mathrm{~mm}$, suitable for a leached flexible fibre bundle endomicroscope probe. Its features include a small diameter and an inflatable balloon to provide a more regular surface for imaging and its ability to scan over a large surface area. The inflated balloon appeared to help stabilize the tissue to some extent, limiting deformation. The achieved mosaicing area was about $30 \mathrm{~mm}^{2}$.

\section{Force control systems}

In soft tissue scanning, since the environment is dynamic and deformable, the scanning motions need to account for tissue deformation to maintain consistency of probe-tissue contact and compression [81]. Some of the force control systems are illustrated in Fig. 5.

Various force control models have been developed for beating heart surgery that have potential to be used in pCLE probe control. For example, Cagneau et al. developed a force control approach using iterative learning control (ILC) that has the advantage of not requiring a model of the contact interaction [82]. The inherent issue of this approach is lack of 


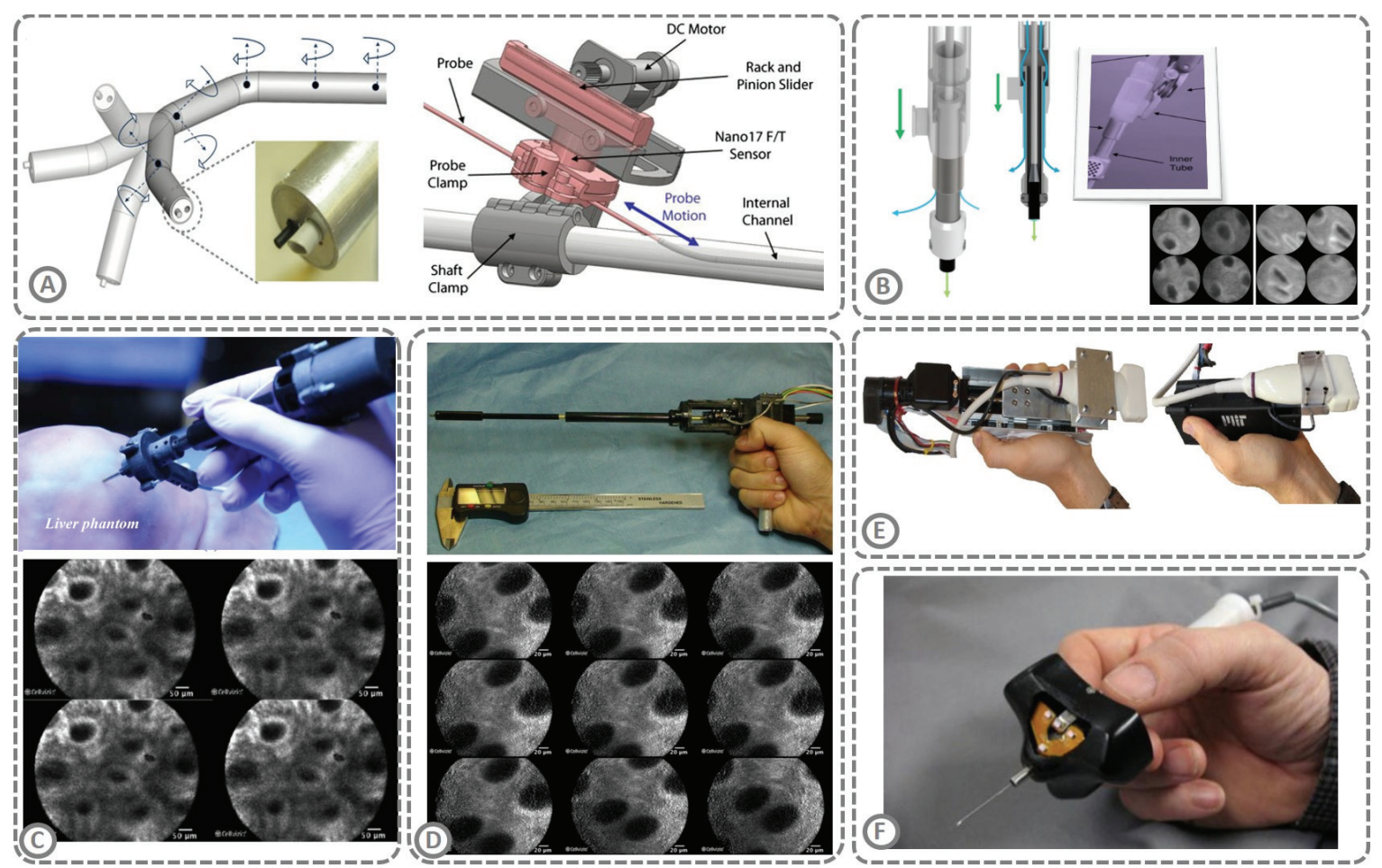

Fig. 5. Force control system, (A) Force adaptive multi-spectral imaging with an embedded 6-axis force/torque sensor (ATI Nano17): adapted from [87], C Springer-Verlag Berlin Heidelberg 2010. All Rights Reserved; (B) A force-contact probe for integration with surgical robot platforms: adapted from [88], (C) Springer-Verlag Berlin Heidelberg 2015. All Rights Reserved; (C) A device incorporating force control for maintaining stable contacts: reprinted with IEEE permission from [89]; (D) Force control device for in vivo deployment: reprinted with IEEE permission from [90]; (E) A hand-held force control system for maintaining consistent contact of an ultrasound imaging probe: reprinted with IEEE permission from [91]; (F) Micron incorporating tremor cancellation by using piezoelectric actuators combined with visual servoing: reprinted with IEEE permission from [94].

robustness. Predictive force control strategy has been proposed by [83-84]. These works have shown acceptable force disturbance rejection and accuracy for beating heart surgery. However, the linear models are adopted for simplicity, which is inappropriate in some cases.

Particularly, the locally weighted projection regression (LWPR) control strategy [85] and impedance control approach [86] are capable of maintaining stable contacts between the targets and probe. LWPR is a function approximator that provides accurate approximation in high dimensional spaces. Using LWPR models can offer an attractive possibility for control design with a higher level of robustness due to online adaptability. However, the LWPR control strategy is still in feasibility test stage. Further developments and improvements are necessary before application in practice. Reference [86] proposes a conventional impedance control law to enable a novel hand-held instrument to apply a constant force over a fast moving target. This strategy reduced the RMS error in the studied bandwidth as much as $60 \%$. Further studies of impedance control will include the implementation of more complicated control strategies to imitate for non-periodic disturbances and time varying dynamic effects.

Noonan et al. combined force adaptive multi-spectral imaging with an embedded 6-axis force/torque sensor (ATI Nano17) which mounted at the proximal end of the imaging probe [87]. A force-contact probe for integration with surgical robot platforms such as the da Vinci was developed by
Giataganas et al. [88]. This paper proposed a force-controlled pick-up probe that can be integrated with the Da Vinci instruments for intraoperative endomicroscopy imaging. The device uses a low-friction air bearing with adaptive axial force control to maintain constant contact between the tissue and the imaging probe, thus facilitating microscopy scans over complex surfaces. Latt et al. developed a device incorporating closed-loop force control for maintaining stable contacts of pCLE probe against tissue [89]. This device was demonstrated to maintain consistent imaging of ex vivo tissue against simulated respiratory motion. They assumed a linear stressstrain relationship of soft tissues for small strains. For simplicity, the soft-tissue is modeled as a linear spring. An enhanced version of this device was developed for in vivo deployment [90]. Gilbertson et al. developed a hand-held force control system for maintaining consistent contact of an ultrasound imaging probe [91]. Actuator travel limits functions were added to prevent overshot of the actuation system. Moreover, this work integrates a tri-axial accelerometer to account for the device's orientation and integrate additional gravity compensation into the force control algorithm.

The endomicroscopy device can also benefit from recent platforms such as the active tremor compensating microsurgical tool (Micron) incorporating tremor cancellation by using piezoelectric actuators combined with visual servoing [92-94]. These devices work by sensing their own motion, and 
then filtering out the components that are considered erroneous motion. The erroneous motion can be cancelled by actuating their surgical end-effectors. A small-signal model of a feedback system with disturbance has been adopted. The small-signal model includes the position servo loop as a component. In the human-in-the-loop system, the manipulation aid converts hand motion into tool motion. Furthermore, they have developed a filter that reduces unintentional motion, yet preserves the intuitive eye-hand coordination.

\section{Haptic feedback and tactile sensing system}

Haptic feedback and tactile sensing is an important topic of ongoing research in both academia and industry, including MIS systems [95, 96], neurosurgery [97] and the endovascular system [98]. For example, reference [98] proposed a master-slave force feedback system for endovascular catheterization that can be used in a natural setting with enhanced ergonomics. Force feedback is based on strain input from the catheter tip deflection. Two independent, PID control loops are used to control the catheter insertion and provide force feedback. The slave system is position controlled and the master system is force controlled. The challenges of deploying haptic technologies in surgical robotics are discussed and reviewed in [99]. As with all MIS, during endomicroscopy procedure, there is a lack of haptic feedback from targeted tissue contact to the operator, which makes it hard to perceive the contact. Haptic feedback is particularly useful in endomicroscopy applications since the soft tissue is very fragile. For hand-held endomicroscopy devices, they allow a manual haptic feedback since they are directly manipulated by the surgeon. Hence, the main role of haptic feedback and tactile sensing in hand-held endomicroscopy devices is to enhance the existing tool-tissue interactions.

A tactile magnification system for the detection of lesions in arthroscopic intervention was developed [100]. The device uses an accelerometer that is integrated into the proximal region of tool to sense the tool-tissue interactions. A filter is used to remove noise, anti-aliased the signal, and enhance system stability. For tactile reproduction, the proposed prototype could amplify the signals by $10 \mathrm{~dB}$ on average. The system could measurably improve users' ability to detect small cuts in cartilage-like elastic surfaces. Research on force sensing based on Fiber-Bragg Gratings [101] may provide haptic information to endomicoscopy robots as well. They customized a micro-forceps by integrating three FBG sensors into the instrument shaft that is typically located in the eye, and can measure a tip force. The disadvantage of this instrument is the lack of axial force sensing. A 3-DOF force sensing instrument is therefore required in future. Payne et al. developed a compact device integrating a three-phase linear motor allow amplification factors of up to $\times 15$. [102]. This device uses a closed-loop PID-based force control scheme to realize the required force amplification. The force measurement of the tool tip provides the feedback to the control loop. The approaches have demonstrated an increase in user sensitivity to tool-tip forces. Stetten et al. proposed a method for amplitude forces perceived by an operator using a tool [103]. A sensor measures the force between the tip of a tool and its handle part by the operator's fingers. These measurements are used to create a magnifying force between the handle and a brace attached to the operator's hand, providing an enhanced tactile between the tip of the tool and a target.

Haptic feedback and tactile sensing will make minimally invasive interventions including endomicroscopy more safe and accurate. In particular, hand-held endomicroscopy devices are suited for such interventions.

\section{FUTURE RESEARCH CHALLENGES}

In recent years, the probe design of endomicroscopy and image mosaicing technologies have seen many improvements, offering practical solutions to real-time large area high-resolution images of tissue micro-structure. The development of steerable technologies has further enhanced the dexterity of mini-probes by providing the precise control of the distal scanning motion [104]. However, their application is limited and further improvements in miniaturization, control and cost of steerable technologies are needed to increase their widespread use. Implementation of force sensing within the devices is a growing research area. Fiber optic technology is one of the solutions for probe force measurement, due to the advantages of small size and compatibility with medical imaging.

Until now, few studies have looked at micro-optical assembly for the tip of the new endomicroscope with consideration for MIS integration and real-time super-resolution enhancement. The design may consider incorporating variable resolution similar to human fovea and parafovea vision but with anisotropic spatial coverage at the periphery. This will create larger effective overlap of the viewing area during pCLE actuation and large area mosaicing, as well as a more realistic prior with controlled transitional/rotational actuation for the proposed real-time super-resolution reconstruction. Micro-optical assembly incorporating fish-eye/cylindrical lenses, diffractive gratings, lenslet arrays and Fresnel lenses are considered as some solutions, especially optical elements with the ability to change their orientation with electro-optic or mechanical means. To enhance the depth resolution of the probe, investigating switchable electro-optic lenses maybe necessary. In addition to optical information, the micro-tip should be designed to incorporate pressure, and force/torque information.

While mosaicing algorithms are developed for large area imaging, these algorithms are still limited in terms of robustness when tissue deformation is encountered. Real-time algorithm need to be developed to enhance the image resolution beyond that of the fibre bundle by utilising multiple views gained from controlled motion of the adaptive micro-optical tip assembly. GPU architecture can be used for to accelerate real-time high fidelity super-resolution algorithms. More specifically, the Split Bregman method [105] enable rapid convergence of general L2 model fitting with L1 optimisation of non-separable prior models. This will enable the use of any multi-scale or gradient based priors. The variable resolution coverage scheme in micro-optical assembly could also provide an underlying map for robust mosaicing to establish dynamic view expansion. Furthermore, 
a periodic sub-pixel rotational trajectory coupled with anisotropic micro-optics could leverage a compressed sensing paradigm to form images surpassing the Nyquist limit, since they introduce a basis in which each super-resolved pixel is supported by a varying multi-scale, multi-orientation field of view in the spatial domain. By using visual servoing to adjust the scanning trajectory and introduce a tip tracking system for correcting mosaicing, it is possible to further enhance the current mosaicing technologies.

Despite recent advances in terms of imaging techniques, most intra-operative imaging relies on 2D mosaicing. In order to realize the promise of endomicroscopy for providing context-aware large area tissue characterization, 3D visualization and navigation are important. Major research issues to be addressed include handling free-form tissue deformation during intervention [106] and correcting co-registration of both pre- and intraoperative data. Framework on inverse realism for real-time intra-operative visualization, for example, can be used [107, 108] and it is possible to incorporate manifold embedding for dimensionality reduction and intuitive tissue contrast enhancement overlaid with contextual imaging information such as pressure and cellular level responses. Furthermore, SLAM framework can be extended with parallel tracking and mapping using groups video frames with pose estimates to generate detailed depth maps via dense matching and triangulation. Additional depth cues from validated 3D real-time tissue deformation recovery methods [109-111] can be used for non-rhythmic deformation in vivo. The fast image mapping system that integrates image mosaics with 3D ultrasound images can be adopted for assisting laser photocoagulation treatment $[112,113]$. Furthermore, for heart beating surgery, the highly sensitive fluorescence endoscope using an electrocardiograph (ECG)-synchronized multiple exposure (ESME) approach could provide a bright fluorescent image [114].

One of the requirements of providing in situ, in vivo imaging beyond the exposed tissue surface is the ability to follow curved anatomical pathways with fully controllable articulation to allow for safe intra-luminal and intra-cavity exploration and intervention. Hand-held robots integrating sensing and actuation can facilitate ease of control for scanning motion. These devices are suitable for endomicroscopy scanning since they are physically smaller and rely on a surgeon's existing dexterity, and thus are lower in cost and requires minimal setup time. The main focus is to develop hand-held multi-DOF mechanisms that investigate the nonlinear effect of changing tortuosity and the use of sensing-based compensation schemes to ensure consistent probe control and force stability. Different proximal/distal mounting arrangement of the sensors and actuation mechanisms need to be assessed with full consideration of the desired miniaturization, probe inter-change and sterilization requirements. It is important to ensure articulation control and tissue targeting are seamlessly coupled with articulation and controlled movement of the micro-optical assembly at the tip for super-resolution.

Due to the complexity of the tissue patterns and interpretation, the imaging date is difficult to be used by inexperienced surgeons. Thus, automated recognition is necessary to help surgeons to identify disease accurately. Hence, it could also reduce the learning curve of inexperienced surgeons. Reference $[115,116]$ discussed a wide range of automated image analysis techniques. The state of the arts on automated nulceus/cell detection and segmentation approaches on digital pathology and microscopy are provided by [117]. Recent developments in deep learning [118-120], machine learning [121-123] and ensemble learning approaches [124-125] have shown good potential to segment the tissue lesions, and analyze the detected tissues.

The application of robotics in endomicroscopy intervention is still in its infancy. Integration of the robotic assisted endomicroscopy with MIS platforms of varying complexities, ranging from the standard endoscopy to robotic platforms (such as the da Vinci [126], fully articulated surgical robot [127] and flexible continuum robot $[128,129])$, is necessary. The integration could include micro-probe optics and packaging; sensing/control and tissue adaptation; real-time image reconstruction, multi-scale fusion, localization and mapping; ergonomic design and instrument/robot navigation. Particular consideration needs to be directed to a self-contained modular design of the probe, allowing inter-changeable use with different MIS platforms and ease of production assembly. Resilience to fatigue and multiple sterilization cycles also needs to be considered. For its use with advanced robotic platforms, additional sensing information from MEMS-based inertial sensors can provide orientation correction during intra-luminal exploration and real-time haptic feedback incorporating dynamic active constraints under large tissue deformation. Ergonomic issues related to the practical control of the device during MIS also need to be explored. Advanced human-robot interaction such as the use of gaze contingent perceptual docking [130, 131] can be used to achieve intuitive targeting and flexible large area surveillance.

\section{CONCLUSIONS}

Microscopic imaging based intervention has revolutionized the fields of histology and MIS over the last two decades. This minimally invasive approach is constantly evolving and now increasingly relies on micro-scale imaging and tissue characterization for precision surgery. The advent of computer and robotic assisted systems designed to address the challenges of endomicroscopy intervention would underpin the future clinical adoption of the technique. These robotic systems enhance dexterity, stability, and precision beyond what is possible with manual techniques. Another area that holds promise for improving manual and robotic endomicroscopy intervention is the development in micro-optics and navigation, including improved tracking of probes, as well as more advanced intra-operative visualization techniques. 3D, real-time tissue visualization, force control and haptic feedback, however, have not been used extensively as yet, and the development of more compact, smart and intuitive handheld robotic endomicroscopy systems hold much promise for the future of endomicroscopy applications, improving patient outcomes as well as reducing costs. 


\section{REFERENCES}

[1] S. Friedland, D. Benaron, S. Coogan, D.Y. Sze, and R. Soetikno, "Diagnosis of chronic mesenteric ischemia by visible light spectroscopy during endoscopy," Gastrointestinal Endoscopy, Vol. 65, pp. 294-300, Feb. 2007.

[2] A. Robichaux-Viehoever, E. Kanter, H. Shappell, D. Billheimer, H. Jones, and A. Mahadevan-Jansen, "Characterization of Raman spectra measured in vivo for the detection of cervical dysplasia," Appl Spectrosc., vol. 61, pp. 986-993, Sep. 2007.

[3] J.A. Jo, Q. Fang, T. Papaioannou, J.D. Baker, A.H. Dorafshar, T. Reil, J.H. Qiao, M.C. Fishbein, J.A. Freischlag, and L. Marcu, "Laguerre-based method for analysis of time-resolved fluorescence data: application to in-vivo characterization and diagnosis of atherosclerotic lesions," Journal of Biomedical Optics, vol. 11, pp. 021004, Mar. 2006.

[4] J.M. Jabbour, M.A. Saldua, J.N. Bixler, and K.C. Maitland, "Confocal Endomicroscopy: Instrumentation and Medical Applications," Ann. Biomed. Eng., vol. 40, pp. 378-397, Feb. 2011.

[5] H. Pohl, T. Rosch, M. Vieth, M. Koch, V. Becker, M. Anders, A.C. Khalifa, and A. Meining, "Miniprobe confocal laser microscopy for the detection of invisible neoplasia in patients with Barrett's oesophagus," Gut, vol.57, pp. 1648-1653, Dec. 2008.

[6] R.C. Newton, S.V. Kemp, G.Z. Yang, A. Darzi, M.N. Sheppard, and P.L. Shah, "Tracheobronchial Amyloidosis and Confocal Endomicroscopy," Respiration, vol.82, no.2, pp. 209-211, Mar. 2011.

[7] R.C. Newton, S. Kemp, Z. Zoumot, G.Z. Yang, A. Darzi, and P.L. Shah, "An unusual case of haemoptysis," Thorax, vol. 65, pp. 796-797, Apr. 2010.

[8] R.C. Newton, S.V. Kemp, P. Shah, D. Elson, A. Darzi, K. Shibuya, S. Mulgrew, and G.Z. Yang, "Progress toward optical biopsy: bringing the microscope to the patient," Lung, vol.189, pp. 111-119, Feb. 2011.

[9] M. Minsky, "Memoir on Inventing the Confocal Scanning Microscope," Scanning vol.10, no.4, pp. 128-138, Jan. 1988.

[10] R. Kiesslich, et al., "Confocal laser endoscopy for diagnosing intraepithelial neoplasias and colorectal cancer in vivo," Gastroenterology vol.127, no.3, pp. 706-713, Sep. 2004.

[11] A.F. Gmitro and D. Aziz, "Confocal microscopy through a fiber-optic imaging bundle," Optics Letters, vol.18, pp. 565-567, Apr. 1993.

[12] G. Le Goualher, A. Perchant, M. Genet, C. Cave, B. Viellerobe, F. Berier, B. Abrat, and N. Ayache, "Towards optical biopsies with an integrated fibered confocal fluorescence microscope," In: Proceedings of the 7th International Conference on Medical Image Computing and Computer-Assisted Intervention, Part II, 2004, pp. 761-768

[13] M. Goetz, "Confocal Laser Endomicroscopy: Applications in Clinical and Translational Science-A Comprehensive Review," ISRN Pathology, Jan. 2012.

[14] C. Pierce, Mark, et al., "Low-cost endomicroscopy in the esophagus and colon," The American journal of gastroenterology vol. 106, no.9, pp. 1722-1724, Sep. 2011

[15] H. Neumann, et al., "Endocytoscopy allows accurate in vivo differentiation of mucosal inflammatory cells in IBD: a pilot study," Inflammatory bowel diseases, vol.19, no.2, pp. 356-362, Feb. 2013.

[16] C.Q. Li et al., "Effects on confocal laser endomicroscopy image quality by different acriflavine concentrations," J Interv Gastroenterol, vol. 1, no. 2, pp. 59-63, Apr. 2011

[17] P. Sharma, et al., "Real-time increased detection of neoplastic tissue in Barrett's esophagus with probe-based confocal laser endomicroscopy: final results of an international multicenter, prospective, randomized, controlled trial," Gastrointest Endosc, vol. 74, pp. 465-472, Sep. 2011.

[18] J.G. Tearney, et al., "In vivo endoscopic optical biopsy with optical coherence tomography," Science, vol. 276, no. 5321, pp. 2037-2039, Jun. 1997

[19] B.J. Vakoc, et al., "Cancer imaging by optical coherence tomography: preclinical progress and clinical potential," Nature Reviews Cancer, vol. 12 , no. 5, pp. 363-368, May 2012

[20] T. Tsung-Han, J.G. Fujimoto, and H. Mashimo, "Endoscopic optical coherence tomography for clinical gastroenterology," Diagnostics, vol. 4, no. 2, pp. 57-93, Jun. 2014

[21] B. E. Bouma, "Evaluation of intracoronary stenting by intravascular optical coherence tomography", Heart, vol. 89, no. 3, pp. 317-320, Mar. 2003

[22] C. Sun, F. Nolte, K. H. Cheng, B. Vuong, K. K. Lee, B. A. Standish, B. Courtney, T. R. Marotta, A. Mariampillai, and V. X.D. Yang, "In vivo feasibility of endovascular doppler optical coherence tomography," Biomed. Opt. Express, vol. 3, pp. 2600-2610, Oct. 2012.
[23] M.J. Gora, et al., "Tethered capsule endomicroscopy enables less invasive imaging of gastrointestinal tract microstructure," Nature medicine, vol. 19, no.2, pp. 238-240, Feb. 2013

[24] M.J. Gora, et al., "Tethered capsule endomicroscopy for comprehensive imaging of the human small intestine," Endoscopic Microscopy X, SPIE BioS 2015, San Francisco, 2015.

[25] http://www.ninepointmedical.com/

[26] https://www.nikoninstruments.com/

[27] http://www.topcon-medical.eu/eu/

[28] M. Bajbouj, M. Vieth, T. Rösch, S. Miehlke, V. Becker, M. Anders, H. Pohl, A. Madisch, T. Schuster, R.M. Schmid, and A. Meining, "Probe-based confocal laser endomicroscopy compared with standard four-quadrant biopsy for evaluation of neoplasia in Barrett's esophagus," Endoscopy, vol. 42, pp. 435-440, Jun. 2010

[29] A. Meining, M. Bajbouj, S. von Delius, and C. Prinz, "Confocal Laser Scanning Microscopy for in vivo Histopathology of the Gastrointestinal Tract," Arab Journal of Gastroenterology, vol. 8, pp. 1-4, 2007

[30] C.S. Loeser, et al., "Confocal endomicroscopic examination of malignant biliary strictures and histologic correlation with lymphatics," J Clin Gastroenterol, vol. 45, no. 3, pp. 246-52, Mar. 2011.

[31] M. Wallace, G. Y. Lauwers, Y. Chen, E. Dekker, P. Fockens, P. Sharma, and A. Meining, "Miami classification for probe-based confocal laser endomicroscopy," Endoscopy, vol. 43, pp. 882-889, Oct. 2011.

[32] R. C. Newton, S. V. Kemp, G. Z. Yang, D. S. Elson, A. Darzi, and P. L. Shah, "Imaging parenchymal lung diseases with confocal endomicroscopy," Respiratory medicine, vol. 106, pp. 127-137, Jan. 2012.

[33] P. Su, et al., "Efficacy of confocal laser endomicroscopy for discriminating colorectal neoplasms from non-neoplasms: a systematic review and meta-analysis," Colorectal Disease, vol. 15, no. 1, pp. e1-e12, Jan. 2013

[34] P.L. Hsiung, J. Hardy, S. Friedland, R. Soetikno, C.B. Du, A.P. Wu, P. Sahbaie, J.M. Crawford, A.W. Lowe, C.H. Contag, and T.D. Wang, "Detection of colonic dysplasia in vivo using a targeted heptapeptide and confocal microendoscopy," Nature Medicine, vol. 14, pp. 454-458, Apr. 2008

[35] M. Goetz, A. Ziebart, S. Foersch, M. Vieth, M.J. Waldner, P. Delaney, P.R. Galle, M.F. Neurath, and R. Kiesslich, "In Vivo Molecular Imaging of Colorectal Cancer With Confocal Endomicroscopy by Targeting Epidermal Growth Factor Receptor," Gastroenterology, vol. 138, pp. 435-446, Feb. 2010

[36] C. Fottner, E. Mettler, M. Goetz, E. Schirrmacher, M. Anlauf, D. Strand, R. Schirrmacher, G. Kloppel, P. Delaney, M. Schreckenberger, P.R. Galle, M.F. Neurath, R. Kiesslich, and M.M. Weber, "In Vivo Molecular Imaging of Somatostatin Receptors in Pancreatic Islet Cells and Neuroendocrine Tumors by Miniaturized Confocal Laser-Scanning Fluorescence Microscopy," Endocrinology, vol. 151, pp. 2179-2188, Feb. 2010

[37] E. Laemmel, M. Genet, G. Le Goualher, A. Perchant, J.-F. Le Gargasson, and E. Vicauta, "Fibered confocal fluorescence microscopy (Cell-viZio ${ }^{\mathrm{TM}}$ ) facilitates extended imaging in the field of microcirculation," Journal of Vascular Research, vol. 41, pp. 400-411, Nov. 2004

[38] S. Abeytunge, Y. Li, B. Larson, G. Peterson, E. Seltzer, R. Toledo-Crow, et al., "Confocal microscopy with strip mosaicing for rapid imaging over large areas of excised tissue," Journal of biomedical optics, vol. 18, pp. 061227 , Jun. 2013.

[39] T.P. Chang, et al., "Imaging of breast cancer morphology using probe-based confocal laser endomicroscopy: Towards a novel imaging tool for real-time intra-operative cavity scanning," European Journal of Surgical Oncology, vol. 39, no. 11, pp. S80-S80, Nov. 2013

[40] T.P. Chang, et al., "Novel classification to facilitate recognition of breast cancer morphology on confocal endomicroscopy," Eur J Surg Oncol., vol. 40, no. 5, pp. 631, May 2014

[41] R. Jeevan, D. Cromwell, M. Trivella, G. Lawrence, O. Kearins, J. Pereira, C. Sheppard, C.M. Caddy, J.H. van der Meulen, "Reoperation rates after breast conserving surgery for breast cancer among women in England: retrospective study of hospital episode statistics," BMJ: British Medical Journal, vol. 345, pp. e4505, Jul. 2012

[42] A. Bogaards, H.J. Sterenborg, J. Trachtenberg, B.C. Wilson, and L. Lilge, "In vivo quantification of fluorescent molecular markers in real-time by ratio imaging for diagnostic screening and image-guided surgery," Lasers in Surgery and Medicine, vol. 39, pp. 605-613, Aug. 2007 
[43] S. Dighe, S. Purkayastha, I. Swift, P.P. Tekkis, A. Darzi, R. A'Herne, and G. Brown, "Diagnostic precision of CT in local staging of colon cancers: a meta-analysis," Clinical Radiology, vol. 65, pp. 708-719, Sep. 2010

[44] A. Bogaards, A. Varma, S.P. Collens, A. Lin, A. Giles, V.X. Yang, J.M. Bilbao, L.D. Lilge, P.J. Muller, and B.C. Wilson, "Increased braintumor resection using fluorescence image guidance in a preclinical model," Lasers in Surgery and Medicine, vol. 35, pp.181-190, Sep. 2004

[45] N. Ayache, T. Vercauteren, G. Malandain, F. Oberrietter, N. Savoire, and A. Perchant, "Processing and mosaicing of fibered confocal images," in Microscopic Image Analysis with Applications in Biology, Oct. 2006.

[46] V. Becker, T. Vercauteren, C.H. von Weyhern, C. Prinz, R.M. Schmid, and A. Meining, "High-resolution miniprobe-based confocal microscopy in combination with video mosaicing (with video)," Gastrointest. Endosc., 66(5), pp.1001-1007, Nov. 2007.

[47] T. Vercauteren, et al, "Robust mosaicing with correction of motion distortions and tissue deformations for in vivo fibered microscopy," Medical image analysis, vol. 10, pp. 673-92, Oct. 2006.

[48] J. Mahé, et al., "A Viterbi approach to topology inference for large scale endomicroscopy video mosaicking," In Medical Image Computing and Computer-Assisted Intervention-MICCAI 2013, 2005, pp. 404-411

[49] N. Bedard, T. Quang, K Schmeler, R. Richards-Kortum, T. Tkaczyk, "Real-time video mosaicing with a high-resolution microendoscope," Biomedical Optics Express, vol. 3, pp. 2428-35, Oct. 2012.

[50] http://www.pentaxmedical.com/pentax/

[51] http://www.maunakeatech.com/en-gb/content/who-are-we

[52] http://www.olympus-global.com/en/medical/

[53] M. K. Quinn, et al., "High-resolution microendoscopy for the detection of cervical neoplasia in low-resource settings," PloS one, vol. 7, no. 9, pp e44924, Sep. 2012.

[54] M. Hughes, et al., "Fiber bundle endocytoscopy," Biomed Opt Express., vol. 4, no. 12, pp. 2781-2794, Nov. 2013.

[55] M. Hughes, P. Giataganas, and G.-Z. Yang, "Color reflectance fiber bundle endomicroscopy without back-reflections," Journal of Biomedical Optics vol. 19, no. 3, pp. 030501- 030501, Mar. 2014.

[56] M. Hughes and G.-Z. Yang, "High speed, line-scanning, fiber bundle fluorescence confocal endomicroscopy for improved mosaicking," Biomedical optics express, vol. 6, no. 4, pp. 1241-1252, Mar. 2015.

[57] J. Sun, C. Shu, B. Appiah, and R. Drezek, "Needle-compatible single fiber bundle image guide reflectance endoscope," Journal of Biomedical Optical Letters, vol. 15, pp. 040502, Jun. 2010.

[58] T. Vercauteren, A. Perchant, X. Pennec, and N. Ayache, "Mosaicing of Confocal Microscopic In Vivo Soft Tissue Video Sequences," International Conference on Medical Image Computing and Computer Assisted Intervention, Lecture Notes in Computer Science, vol. 3749, Feb. 2005, pp. 753-760.

[59] T. Vercauteren, X. Pennec, A. Perchant, and N. Ayache, "Diffeomorphic Demons: Efficient Non-parametric Image Registration," NeuroImage, vol. 45, pp. S61-72, Nov. 2009.

[60] B. Rosa, et al., "Building large mosaics of confocal endomicroscopic images using visual servoing," IEEE transactions on biomedical engineering, vol. 60, pp. 1041-1049, Nov. 2013.

[61] K. Vyas, M. Hughes, and G.-Z. Yang, "Electromagnetic tracking of handheld high-resolution endomicroscopy probes to assist with real-time video mosaicking," SPIE BiOS, International Society for Optics and Photonics, Mar. 2015.

[62] T. Vercauteren, et al., "Real time autonomous video image registration for endomicroscopy: fighting the compromises," In Biomedical Optics (BiOS), Feb. 2008, pp. 68610C-68610C

[63] P. Mountney, S. Giannarou, D. Elson, and G.-Z. Yang, "Optical Biopsy Mapping for Minimally Invasive Cancer Screening," International Conference on Medical Image Computing and Computer Aided Intervention, Lecture Notes in Computer Science, vol. 5761, Sep. 2009, pp. 483-490.

[64] G. Dwyer, P. Giataganas, P. Pratt, M. Hughes, and G.-Z. Yang, “A miniaturised robotic probe for real-time intraoperative fusion of ultrasound and endomicroscopy," In proc. IEEE Int. Conf. Robotics Automation, May. 2015, pp. 1196-1201.

[65] J. Yang, , J. Wright, T. Huang, and M. Yi, "Image super-resolution as sparse representation of raw image patches," IEEE Conference on Computer Vision and Pattern Recognition, Anchorage, AL, Jun. 2008, pp. $1-8$.

[66] M. Elad, and M. Zibulevsky, "Iterative shrinkage algorithms and their acceleration for L1-L2 signal and image processing applications," IEEE Signal Processing Magazine, vol. 27, pp. 78-88, 2010.
[67] R. Molina, M. Vega, J. Mateos, and A.K. Katsaggelos, "Variational posterior distribution approximation in Bayesian super resolution reconstruction of multispectral images," Applied and Computational Harmonic Analysis ,vol. 24, pp. 251-267, Nov. 2007.

[68] L. C. Pickup, D.P. Capel, S.J. Roberts, and A. Zisserman, "Bayesian Methods for Image Super-Resolution,” The Computer Journal, vol. 52, pp. 101-113, Jan. 2009.

[69] A. W. Dowsey, and G.-Z. Yang, "Automatic alignment, statistical restoration and quantification of raw LC/MS and 2-DE data," 8th Annual HUPO World Congress, 2009, pp. C523

[70] S. Rupp, M. Elter, and C. Winter, "Improving the Accuracy of Feature Extraction for Flexible Endoscope Calibration by Spatial Super Resolution," 29th Annual International Conference of the IEEE Engineering in Medicine and Biology Society, Aug. 2007, pp. 6565-6571.

[71] S. Farsiu, M. Elad, and P. Milanfar, "Video-to-Video Dynamic Super-Resolution for Grayscale and Color Sequences," EURASIP Journal on Applied Signal Processing, pp.1-15, Jan. 2006.

[72] P. Giataganas, et al., "Cooperative in situ microscopic scanning and simultaneous tissue surface reconstruction using a compliant robotic manipulator,' In proc. IEEE Int. Conf. Robotics Automation, May 2013, pp. $5378-5383$.

[73] M. S. Erden, B. Rosa, J. Szewczyk, and G. Morel, "Mechanical design of a distal scanner for confocal microlaparoscope:Aconic solution," IEEE Int. Conf. Robot. Autom, May 2013, pp. 1205-1212.

[74] D. P. Noonan, D. S. Elson, G. P. Mylonas, A. Darzi, and G. Z. Yang, "Laser-induced fluorescence and reflected white light imaging for robot-assisted MIS," IEEE transactions on biomedical engineering, vol. 56, pp. 889-92, Nov. 2008.

[75] R. Newton, D. Noonan, V. Vitiello, J. Clark, C. Payne, J. Shang, M. Sodergren, A. Darzi and G.-Z. Yang, "Robot-assisted transvaginal peritoneoscopy using confocal endomicroscopy: A feasibility study in a porcine model," Surgical Endoscopy, vol. 26, pp. 2532-2540, Apr. 2012.

[76] B. Rosa, B. Herman, J. Szewczyk, B. Gayet, and G. Morel, "Laparoscopic optical biopsies: in vivo robotized mosaicing with probe-based confocal endomicroscopy," in Proc. of IROS'2011, San Francisco, California, Sep. 2011, pp.1339-1345.

[77] M. S. Erden, et al., "Conic-Spiraleur: A Miniature Distal Scanner for Confocal Microlaparoscope," IEEE/ASME transactions on mechatronics, vol. 19no. 6, pp. 1786 - 1798, Dec. 2014.

[78] S. Zuo et al., "Development of a Large Area Scanner for Intraoperative Breast Endomicroscopy," Proc. IEEE Int. Conf. Robotics Automation, May 2014, pp. 3524 - 3530.

[79] S. Zuo, M. Hughes, C. Seneci, T.P. Chang, and G.-Z. Yang, "Towards Intraoperative Breast Endomicroscopy with a Novel Surface Scanning Device,” IEEE Trans. Biomed. Eng. Vol.62, no. 12, pp. 2941-2952, Dec. 2015.

[80] S. Zuo, M. Hughes, and G.-Z. Yang, "Novel Balloon Surface Scanning Device for Intraoperative Breast Endomicroscopy," Annals of biomedical engineering, vol. 44, no.7, pp. 2313-2326, Oct. 2015.

[81] R. C. Newton et al., "Probe tip contact force and bowel distension affect crypt morphology during confocal endomicroscopy," Gut, vol. 60, pp.A12 -A13, Mar. 2011.

[82] B. Cagneau, N. Zemiti, D. Bellot, and G. Morel, "Physiological motion compensation in robotized surgery using force feedback control," In: IEEE International Conference on Robotics and Automation, Apr. 2007, pp. $1881-1886$

[83] M. Dominici, P. Poignet, R. Cortes ao, E. Dombre, and O. Tempier, "Compensation for $3 \mathrm{~d}$ physiological motion in robotic-assisted surgery using a predictive force controller: experimental results," In: IEEE/RSJ international conference on Intelligent robots and systems, Piscataway, NJ, USA: IEEE Press, 2009, pp. 2634-2639.

[84] S. G. Yuen, M. C. Yip, N. V. Vasilyev, D. P. Perrin, P. J. del Nido, and R. D. Howe, "Robotic force stabilization for beating heart intracardiac surgery," in MICCAI (1), 2009, pp. 26-33.

[85] J.M. Florez, D. Bellot, and G. Morel, "LWPR-model based predictive force control for serial comanipulation in beating heart surgery," In: IEEE/ASME International Conference on Advanced Intelligent Mechatronics, 2011, pp. 320-326.

[86] J.M. Florez, J. Szewczyk, and G. Morel, "An impedance control strategy for a hand-held instrument to compensate for physiological motion," In: IEEE International Conference on Robotics and Automation, 2012, pp. 1952-1957.

[87] D. Noonan, , C. Payne, J. Shang, V. Sauvage, R. Newton, D. Elson, A. Darzi, and G.-Z. Yang, "Force Adaptive Multi-spectral Imaging with an 
Articulated Robotic Endoscope," Medical Image Computing and Computer-Assisted Intervention, vol. 3, Sep. 2010, pp. 245-252

[88] P. Giataganas, M. Hughes and G.-Z. Yang, "Force adaptive robotically assisted endomicroscopy for intraoperative tumour identification," International journal of computer assisted radiology and surgery, vol.10, no.6, pp. 1-8, Apr. 2015

[89] W. T. Latt, R. C. Newton, M. Visentini-Scarzanella, C. J. Payne, D. P. Noonan, J. Shang, and G.-Z. Yang, "A handheld instrument to maintain steady tissue contact during probe-based confocal laser endomicroscopy," IEEE Trans. Biomed. Eng. Vol. 58, pp. 2694-2703, Sep. 2011

[90] W. T. Latt, T. P. Chang, A. Di Marco, P. Pratt, K.-W. Kwok, J. Clark, and G.-Z. Yang, "A hand-held instrument for in vivo probe-based confocal laser endomicroscopy during minimally invasive surgery," In: IEEE/RSJ International Conference on Intelligent Robots and Systems, Oct. 2012, pp. 1982-1987.

[91] M. W. Gilbertson, and B. W. Anthony, "Ergonomic control strategies for a handheld force-controlled ultrasound probe," In: IEEE/RSJ International Conference on Intelligent Robots and Systems, 1, Oct. 2012, pp. 1284-129

[92] W.T. Ang, C.N. Riviere, P.K. Khosla, "An Active Hand-held Instrument for Enhanced Microsurgical Accuracy," International Conference on Medical Image Computing and Computer Assisted Intervention, Pittsburgh, PA, Lecture Notes in Computer Science,vol.1935, Apr. 2001, pp. 243-291

[93] B.C. Becker, , C.R. Valdivieso, J. Biswas, L.A. Lobes, C.N. Riviere, "Active guidance for laser retinal surgery with a handheld instrument," 31 st Annual International Conference of the IEEE Engineering in Medicine and Biology Society, Minneapolis, MN, Sep. 2009, pp.339-344.

[94] R. A. Maclachlan , B. C. Becker, J. C. Tabare' s, G. W. Podnar, L. A. Lobes, and C. N. Riviere, "Micron: an actively stabilized handheld tool for microsurgery," IEEE Trans. Robot., vol. 28,pp. 195-212, Nov. 2012.

[95] G. Tholey , J. P. Desai, and A. E. Castellanos, "Force feedback plays a significant role in minimally invasive surgery: results and analysis," Ann Surg, vol. 241, pp.102-109, Jan. 2005.

[96] B. Kuebler, U. Seibold, and G. Hirzinger, "Development of actuated and sensor integrated forceps for minimally invasive robotic surgery," Int. J. Med. Robot. Comput, Assist. Surg, vol. 1, pp.96-107, Sep. 2005.

[97] G. R. Sutherland, P. B. McBeth, and D. F. Louw, "Neuroarm: an MR compatible robot for microsurgery," In: Computer Assisted Radiology and Surgery, pp. 504-508, Jun. 2003.

[98] C. J. Payne, H. Rafii-Tari, and G.-Z. Yang, "A force feedback system for endovascular catheterisation," In: IEEE/RSJ International Conference on Intelligent Robots and Systems, Oct. 2012, pp. 1298-1304.

[99] N. Enayati, E.M. Momi, G. Ferrigno, "Haptics in robot-assisted surgery: challenges and benefits," IEEE Rev Biomed Eng., vol. 9, pp. 49-65, 2016.

[100]H.-Y. Yao, V. Hayward, and R. E. Ellis, "A tactile enhancement instrument for minimally invasive surgery," Comput. Aided. Surg, vol.10, pp.233-239, Aug. 2005.

[101]X. He, M. A. Balicki, J. U. Kang, P. L. Gehlbach, J. T. Handa, R. H. Taylor, and I. I. Iordachita, "Force sensing micro-forceps with integrated fiber Bragg grating for vitreoretinal surgery," SPIE BiOS Int. Soc. Optics. Photonics, Feb. 2012, pp.82180W-82180W.

[102]C. J. Payne, W. T. Latt, and G.-Z. Yang, "A new hand-held force-amplifying device for micromanipulation," In: IEEE International Conference on Robotics and Automation, May 2012, pp. 1583-1588.

[103]G. Stetten, B. Wu, R. Klatzky et al., "Hand-held force magnifier for surgical instruments," In: Augmented Environments for Computer-Assisted Interventions, Jun. 2011, pp. 90- 100.

[104]H. Yamashita, et al., "Miniature bending manipulator for fetoscopic intrauterine laser therapy to treat twin-to-twin transfusion syndrome," Surgical Endoscopy, Vol. 22 No. 2, pp. 430-435, 2008.

[105]T. Goldstein, and S. Osher, "The Split Bregman Method for L1-Regularized Problems," SIAM Journal on Imaging Sciences, vol. 2, pp. 323-343, Apr. 2009.

[106]M. Lerotic, A.J. Chung, J. Clark, S. Valibeik, G.-Z. Yang, "Dynamic View Expansion for Enhanced Navigation in Natural Orifice Transluminal Endoscopic Surgery," International Conference on Medical Image Computing and Computer Aided Intervention, Brisbane, Australia, Lecture Notes in Computer Science, vol. 5242, Sep. 2007, pp. 467-475.

[107]P. Pratt, E. Mayer, J. Vale, D. Cohen, E. Edwards, A. Darzi and G.-Z. Yang, "An effective visualisation and registration system for image-guided robotic partial nephrectomy," J. Robotic Surgery, vol. 6, pp. 23-31, Mar. 2012

[108]K. Sun, T. S. Pheiffer, A. L. Simpson, J. A. Weis, R. C. Thompson and M. I. Miga, "Near real-time computer assisted surgery for brain shift correction using biomechanical models," IEEE J. Translational Eng. Health Med., vol. 2, Apr. 2014.

[109]D. Stoyanov, and G.-Z. Yang, "Stabilization of Image Motion for Robotic Assisted Beating Heart Surgery," International Conference on Medical Image Computing and Computer Assisted Interventions, Brisbane, Australia, Lecture Notes in Computer Science, vol. 4791, Feb. 2007, pp. 417-424.

[110]D. Stoyanov, G.P. Mylonas, M. Lerotic, A.J. Chung, and G.-Z. Yang, "Intra-Operative Visualizations: Perceptual Fidelity and Human Factors," Journal of Display Technology, vol. 4, pp. 491-501, Jan. 2009.

[111]D. Stoyanov, M. Visentini-Scarzanella, P. Pratt, G.-Z. Yang, "Real-time Stereo Reconstruction in Robotic Assisted Minimally Invasive Surgery," Medical Image Computing and Computer-Assisted Intervention, vol. 13, no.1, Sep. 2010, pp. 275-282.

[112]H. Liao, et al., "Fast Image Mapping of Endoscopic Image Mosaics with Three-Dimensional Ultrasound Image for Intrauterine Fetal Surgery," Minimally Invasive Therapy and Allied Technologies, Vol. 18, No. 6, pp. 332-340, 2009.

[113]L. Yang, et al., "Ultrasound image-guided mapping of endoscopic views on 3D placenta model: a tracker-less approach," The 6th International Workshop on Medical Imaging and Augmented Reality, MIAR 2013, LNCS 8090, 2013, pp. 107-116

[114]T. Ando, et al., "High Sensitive Fluorescence Endoscope using Electrocardiography-Synchronized Multiple Exposure," International Journal of Computer Assisted Radiology and Surgery, Vol. 6, No. 1, pp. 73-81, 2011

[115]A.A. Abder-Rahman and T. M. Deserno, "A systematic review of automated melanoma detection in dermatoscopic images and its ground truth data," In: Proc. SPIE, Medical Imaging 2012: Image Perception, Observer Performance, and Technology Assessment, vol. 8318, 2012.

[116]V. Stoecker, N. Mishra, R. LeAnder, R. Rader, and R. Stanley. "Automatic Detection of Skin Cancer - Current Status, Path for the Future," In: Proceedings of the International Conference on Computer Vision Theory and Applications, 2013, pp. 504-508.

[117]F. Xing, L. Yang, "Robust nuclei/cell detection and segmentation for digital pathology and microscopic images: a comprehensive review," IEEE Rev Biomed Eng, vol. 9, pp. 234-263, 2016.

[118]N. Codella, J. Cai, M. Abedini, R. Garnavi, A. Halpern, and J. Smith. "Deep Learning, Sparse Coding, and SVM for Melanoma Recognition in Dermoscopy Images," Proceedings of the 6th International Workshop on Machine Learning in Medical Imaging (MLMI), LNCS 9352, 2015, pp. 118-126.

[119]K. He, X. Zhang, S. Ren, and J. Sun, "Deep Residual Learning for Image Recognition," IEEE Conference on Computer Vision and Pattern Recognition (CVPR), 2016, pp. 770-778.

[120]N. C. F. Codella, Q. B. Nguyen, S. Pankanti, D. Gutman, B. Helba, A. Halpern, and J. R. Smith, "Deep learning ensembles for melanoma recognition in dermoscopy images," IBM Journal of Research and Development, vol. 61, no. 4/5, 2017

[121]F. Ercal, A. Chawla, W. V. Stoecker, Hsi-Chieh Lee, and R. H. Moss, "Neural network diagnosis of malignant melanoma from color images," in IEEE Transactions on Biomedical Engineering, vol. 41, no. 9, pp. 837-845, Sept. 1994.

[122] C. Barata, M. Ruela, M. Francisco, T. Mendonça, J. S. Marques "Two Systems for the Detection of Melanomas in Dermoscopy Images using Texture and Color Features," IEEE Systems Journal, vol. 99, pp. 1-15, 2014.

[123]J. A. Jaleel, S. Salim, R.B. Aswin, "Artificial Neural Network Based Detection of Skin Cancer," in International Journal of Advanced Research in Electrical, Electronics and Instrumentation Engineering, vol. 1, no. 3, pp. 200-205, 2012.

[124]T. F. Mendonca, P. M. Ferreira, A. R. S. Marcal, C. Barata, J. S. Marques, J. Rocha, and J. Rozeira, "PH2: A Public Database for the Analysis of Dermoscopic Images," in Dermoscopy Image Analysis (M. E. Celebi, T. Mendonca, and J. S. Marques, eds.), pp. 419-439, CRC Press, 2015.

[125]G, Schaefer, B. Krawczyk, M. E. Celebi, and H. Iyatomi, "An ensemble classification approach for melanoma diagnosis," in Memetic Computing, vol. 6, no. 4, pp. 233-240, 2014

[126]A. Carpentier, D. Loulmet, B. Aupecle, A. Berrebi, and J. Rellard, "Computer-assisted cardiac surgery," Lancet, vol. 353, pp. 379-380, Jan. 1999. 
[127]J. Shang et al., "An articulated universal joint based flexible access robot for minimally invasive surgery," In Proc. IEEE Int. Conf. Robotics Automation, Jun. 2011, pp. 1147-1152.

[128]S. Zuo et al., "Variable stiffness outer sheath with "Dragon skin" structure and negative pneumatic shape-locking", International Journal of Computer Assisted Radiology and Surgery, vol. 9, issue. 5, pp. 857-865, Sep. 2014

[129]S. Zuo, N. Yamanaka, I. Sato, K. Masamune, H. Liao, K. Matsumiya, and T. Dohi, "Mri-compatible rigid and flexible outer sheath device with pneumatic locking mechanism for minimally invasive surgery," in Proc. 4th Int. Workshop Med. Imag. Augmented Reality, Tokyo, Japan, Aug. 1-2, 2008, vol. 5128, pp. 210-219.

[130]G.-Z. Yang, G.P. Mylonas, K.-W. Kwok, and A. Chung, "Perceptual Docking for Robotic Control," 4th International Workshop on Medical Imaging and Augmented Reality, Tokyo, Japan, Lecture Notes in Computer Science, vol. 5128, Aug. 2008, pp. 21-30.

[131]G.P Mylonas, K. Kwok, D. James, D. Leff, F. Orihuela-Espina, A. Darzi, and G.-Z. Yang, "Gaze-Contingent Motor Channelling, Haptic Constraints and associated Cognitive Demand for Robotic MIS," Medical Image Analysis vol. 16, no. 3, pp. 612-631, Aug. 2010.

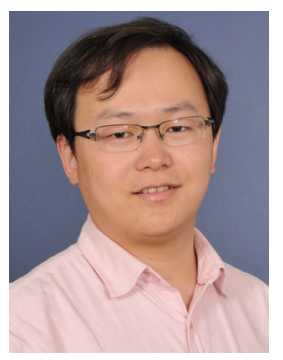

Siyang Zuo received the M.Eng. and Ph.D. degrees from the University of Tokyo, Japan, in 2009 and 2013, respectively. He is currently a Full Professor in the Key Laboratory of Mechanism Theory and Equipment Design of Ministry of Education, School of Mechanical Engineering, Tianjin University, China. His research interests include medical robotics and instruments, computer assisted surgical systems, and medical imaging techniques. $\mathrm{He}$ is distinguished by selection for "National Thousand Young Talents" Program (National Recruitment Program of Global Experts, China). He is also the recipient of various awards including Young Scientist Award (2011) presented by the 14th International Conference on Medical Image Computing and Computer Assisted Intervention (MICCAI2011), and Best Engineering Paper Award (2013) presented by Journal of Japan Society of Computer Aided Surgery.

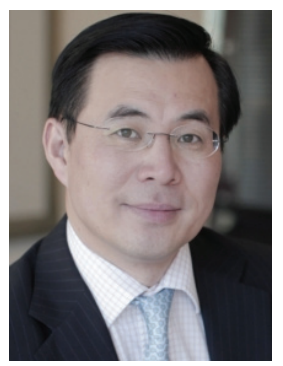

Guang-Zhong Yang (S'90-M'91-SM'08-F'11) received his $\mathrm{PhD}$ in Computer Science from Imperial College London, U.K. He is the director, co-founder of the Hamlyn Centre for Robotic Surgery, Deputy Chairman of the Institute of Global Health Innovation. Professor Yang also holds a number of key academic positions at Imperial College - he is director and founder of the Royal Society/Wolfson Medical Image Computing Laboratory, co-founder of the Wolfson Surgical Technology Laboratory, and chairman of the Centre for Pervasive Sensing. He is also a Distinguished Lecturer for IEEE Engineering in Medicine and Biology Society (EMBS) and a Fellow of the Royal Academy of Engineering (RAEng), the Institute of Electrical and Electronics Engineers (IEEE), the Institution of Engineering and Technology (IET), the American Institute for Medical and Biological Engineering (AIMBE), the International Academy of Medical and Biological Engineering (IAMBE), the Society of Medical Imaging and Computer Assisted Intervention (MICCAI), and the City and Guilds of London. He is equally a recipient of the Royal Society Research Merit Award, the I.I. Rabi Award from the International Society for Magnetic Resonance in Medicine and The Times Eureka 'Top 100' in British Science. He is also editor-in-chief of prestigious journals in the fields of health informatics and robotics. He pioneered the concept of perceptual docking for robotic control as well as the field of Body Sensor Networks (BSN). Professor Yang's main research interests are in medical imaging, sensing and robotics. 